\title{
Supporting Information for: \\ Rapid Method for Calculating the Conformationally Averaged Electronic Structure of Conjugated Polymers
}

Rex Manurung, Ping Li ${ }^{\dagger}$ and Alessandro Troisi*

University of Liverpool, Crown St, Liverpool L69 7ZD

'Current Address: Nanjing University of Posts and Telecommunications, 9 Wenyuan Road, Nanjing 210023, China

*E-mail: a.troisi@liverpool.ac.uk

This supporting information contains:

S1. Method for calculating matrices in the localised molecular orbital basis (pg. 2-3)

S2. Relaxed dimer structure calculation details (pg. 4)

S3. Calibration method and results (pg. 4-5)

S4. Density of states (DOS) calculation and error in the DOS post-calibration (pg. 6-7)

S5. Calculation of localisation length and inverse participation ratio (pg. 7-8)

S6. Torsional energy vs dihedral angle (pg. 9-10)

S7. Tail slope comparisons and reduced $\pi$-basis set LMOM calculations (pg. 10-11) 


\section{S1. Method for calculating matrices in the localised molecular orbital basis}

The localised molecular orbital (LMO) basis matrices are obtained by first calculating the corresponding atomic basis Fock and overlap matrices $\mathbf{F}^{\mathbf{A}}$ and $\mathbf{S}^{\mathbf{A}}$ and then transforming these using molecular orbitals with coefficients expressed in the LMO basis. $\mathbf{F}^{\mathbf{A}}$ and $\mathbf{S}^{\mathbf{A}}$ are obtained by calculating them for hydrogen capped equivalents of the monomer and dimer fragments. The basis transform from atomic to LMO basis is given for the Fock and overlap matrices by equations SE1 and SE2, respectively, where the LMO basis elements thus obtained are $F_{k i, j l}^{\mathrm{LMO}}$ and $S_{k i, j l}^{\mathrm{LMO}}$.

$F_{k i, j l}^{\mathrm{LMO}}=\mathbf{C}_{k i}^{\mathrm{T}} \mathbf{F}^{\mathrm{A}} \mathbf{C}_{j l}$

$S_{k i, j l}^{\mathrm{LMO}}=\mathbf{C}_{k i}^{\mathrm{T}} \mathbf{S}^{\mathbf{A}} \mathbf{C}_{j l}$

The row vector $\mathbf{C}_{k i}$ is the $i$ th $\mathrm{MO}$ on the $k$ th monomer in the polymer chain expressed in the LMO basis. This is done by truncating $\mathbf{C}_{k i}$ to contain coefficients only from basis functions that exist in the fragment with atomic basis matrices $\mathbf{F}^{\mathbf{A}}$ and $\mathbf{S}^{\mathbf{A}}$. If the fragment is a monomer $\mathbf{C}_{k i}$ is unchanged. For a dimer fragment $\mathbf{C}_{k i}$ is expressed in the LMO basis by including only the basis function coefficients of the $i$ th $\mathrm{MO}$ on the $k$ th monomer which are also present in the dimer, and expanding the number of coefficients with 0 s to match the size of the dimer basis. The MOs $\mathbf{C}_{k i}$ are obtained by calculating them for a rigid monomer in the relative geometry they would occupy in the dimer fragment.

For dimer fragments the signs of the monomer $\pi-\mathrm{MO}$ coefficients $\mathbf{C}_{k i}$ are first corrected to ensure they are consistent with the orientation of the monomers in the dimer before using $\mathbf{C}_{k i}$ in SE1 and SE2. This is done by forcing the phases of the p-orbitals in the $\pi$-MOs to align with the direction of a reference axis $\mathbf{d}_{\mathbf{h}}$ which is perpendicular to the monomer plane. $\mathbf{d}_{\mathbf{h}}$ is calculated by selecting three atoms involved in the $\pi$-conjugation on each monomer and calculating the cross product SE3 of the distance vectors $\mathbf{d}_{\mathbf{1}}$ and $\mathbf{d}_{2}$, defined by the position vectors $\mathbf{r}_{1}, \mathbf{r}_{2}$, and $\mathbf{r}_{3}$ corresponding to cartesian coordinates of the three atoms selected, labelled 1, 2 and 3 in figure S1.

$d_{h}=d_{1} \times d_{2}=\left(r_{1}-r_{2}\right) \times\left(r_{3}-r_{2}\right)$

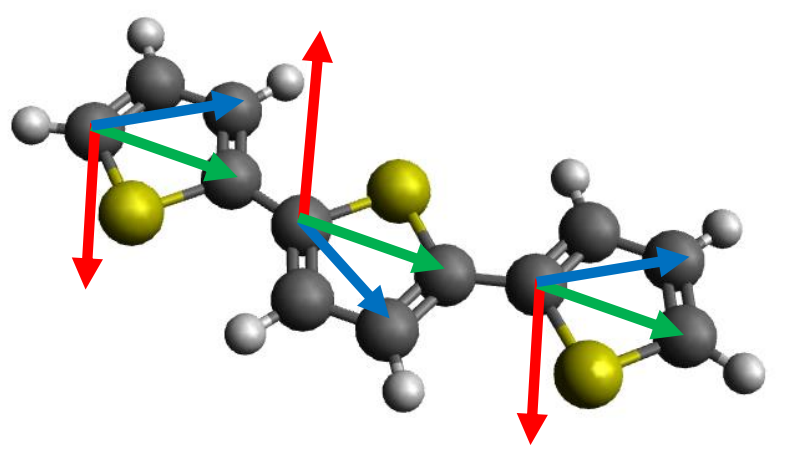

Figure S1: Illustration of how vectors $\mathbf{d}_{1}$ (blue) and $\mathbf{d}_{\mathbf{2}}$ (green) would be defined on individual monomers in a polymer chain, and the vector $\mathbf{d}_{h}\left(\right.$ red) obtained from the cross product of $\mathbf{d}_{\mathbf{1}}$ and $\mathbf{d}_{\mathbf{2}}$ on each monomer.

The convention is to choose atoms 2 and 3 to be the ones connected to adjacent monomers, and atom 1 can be chosen as any other carbon atom involved in the $\pi$-conjugation. $A$ vector $\mathbf{W}$ is then constructed containing the values of the $2 p_{x}, 2 p_{y}$ and $2 p_{z}$ coefficients on the LMO $\mathbf{C}_{k i}$ at atom 2 , given in equation SE4. The sign of the dot product $\mathbf{W}$ and $\mathbf{d}_{\mathbf{h}}$ is used as a sign correction factor $g$ in equation SE5 which is multiplied with all the coefficients of the LMO $\mathbf{C}_{k i}$. The p-orbital phases on $\mathbf{C}_{k i}$ 
are only changed when $g=-1$ thus equation SE5 aligns these $p$-orbital phases in the positive direction of $\mathbf{d}_{\mathbf{h}}$ or leaves them unchanged when $g=1$.

$\mathbf{W}=\left[\begin{array}{lll}2 p_{x} & 2 p_{y} & 2 p_{z}\end{array}\right]$

$g=\operatorname{sgn}\left(\mathbf{W} \cdot \mathbf{d}_{\mathbf{h}}\right)$

The second correction done for the LMO basis matrices fixes the signs of the LMO basis coupling elements for $A-B$ type dimers, where $A \neq B$. This is required as the sign of the coupling between any two MOs of the 2 monomers in an A-B dimer can change due to the symmetry of $\pi$-orbitals. In monomers where there are chemically identical connection sites, the difference in the sign of MO couplings between 2 adjacent monomers depends on the order in which these monomers occur in the polymer chain (A-B or B-A order), illustrated in figure S2. This occurs as the orbital phases of the p-orbitals in the MOs are treated as being fixed to the orientation of the monomer geometry.

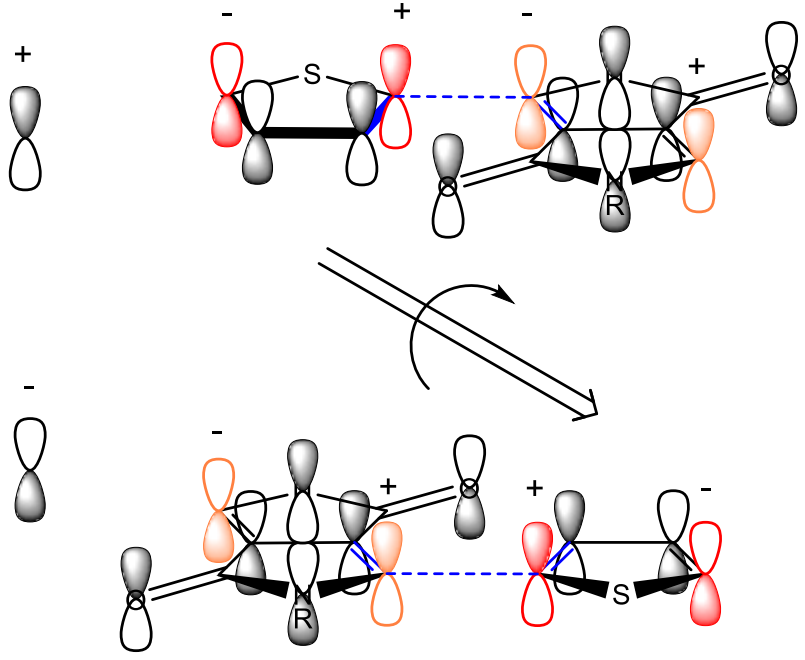

Figure S2: Illustration of the effect of changing the order of dimers along a chain from A-B to B-A permutation while fixing the phase of the $p$-orbitals on each monomer and dihedral angle.

The second correction to the coupling element signs considers the p-orbital at the connection site atom of a monomer is the one lying perpendicular to the $\sigma$-bonds, which is taken here as the $2 p_{y}$ component. The $2 p_{y}$ orbital on each connection site atom is assigned a sign, -1 or +1 , which is determined by the phase pattern of the p-orbital lobes (figure S2). As we only consider linear polymer chains, each monomer thus has only 2 possible connection sites, which may be chemically identical or distinct. If at least one of monomers $A$ and $B$ in the dimer A-B has chemically identical connection sites, the sign of the coupling for any 2 LMOs is changed according to table S1 to obtain the coupling for those same LMOs in the dimer pair B-A.

\begin{tabular}{l|l}
\hline Table S1 - MO coefficient sign modification table \\
\hline $\begin{array}{l}\text { Signs of } 2 p_{y} \text { atomic orbital on } \\
\text { connection site atoms in } \\
\text { monomer A (right) to B (left) }\end{array}$ & $\begin{array}{l}\text { Factor multiplied to sign of coupling element for } 2 \text { LMOs in } \\
\text { the dimer pair A-B to obtain corresponding element for the } \\
\text { same } 2 \text { LMOs in permutation B-A }\end{array}$ \\
\hline$+1,+1$ & None \\
\hline$-1,+1$ & Coupling element is multiplied by -1 for permutation B-A \\
\hline$-1,-1$ & None \\
\hline
\end{tabular}




\section{S2. Relaxed dimer structure calculation details}

Relaxed torsional scan calculations were done at B3LYP/6-311G++** for dimers containing the monomers in the sample shown in figure S3. These monomers are the entire sample needed to build chains of our 5 polymers IDTBT, DPPBTz, DPPDTT, polythiophene and PDPP2TT using the scheme outlined in section 2.2 of the main paper.
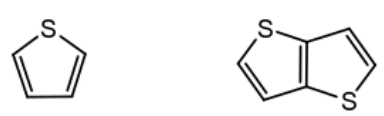

$\mathrm{T}$

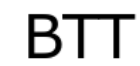<smiles>O=C1NC=C2CNC(=O)C12</smiles>

DPP

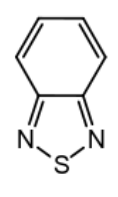

BT

Figure S3: The sample of monomers with which dimers were generated. Torsional scans were calculated for dimers of these monomers.

For our 5 polymers IDTBT, DPPBTz, DPPDTT, polythiophene and PDPP2TT, the benzotriazole monomer BTz of DPPBTz and IDT monomer of IDTBT were not included in the sample of figure S3. The analogues for these were chosen to be benzothiadazole (BT) and thiophene ( $T$ ), respectively. Dimer analogues for these for monomer pairs occurring in the polymers were therefore IDT-BT $=T-B T, T Z-T=B T-T$, and Tz-DPP = BT-DPP.

\section{S3. Calibration method and results}

The linear and polynomial regression fitting used for calibration of the LMOM energies are described in equations SE6, SE7 and SE8. From the linear regression fit, parameters $m$ and $c$ are used to obtain the calibrated LMOM energies $E_{\text {LMOM }}^{\prime}$ from the original LMOM energy $E_{L M O M}$.

$E_{L M O M}^{\prime}=\frac{E_{L M O M}-c}{m}$

For a polynomial regression curve fit, a trial function in equation SE8 is used to obtain the fitting parameters $u, v$ and $w$. These parameters are obtained by fitting the LMOM energies, $x$, to the exact energies $y$ using polynomial regression up to the $2^{\text {nd }}$ order term. The parameters $u, v$, and $w$ are then used in equation SE8 to calculate the calibrated LMOM energies $E_{L M O M}^{\prime}$ from the original LMOM energy $E_{L M O M}$.

$y=u x^{2}+v x+w$

$E_{L M O M}^{\prime}=u E_{L M O M}{ }^{2}+v E_{L M O M}+w$

The accuracy of the calibration schemes was measured using the root mean square error (RMSE) defined in equation SE9, where $\mathrm{N}$ is the number of chains in the sample.

$R M S E_{f i t}=\sqrt{\frac{\left(E^{\prime}{ }_{L M O M}-E_{F C}\right)^{2}}{N}}$

The values for $R M S E_{f i t}$ were calculated for each calibration sample size (5, 25, 50 and 100 chains) and chain length $(6,12$ and 24), with these being some multiple of 100 chains and 48 monomers, respectively, and for both linear and curve-based calibration methods (tables S2 and S3). 
Table S2: RMSE values with polynomial and line regression fits for polythiophene against time cost of generating the calibration sample (chain length=calibration chain length, number of chains=calibration sample size)

\begin{tabular}{|c|c|c|c|c|c|c|c|c|c|c|c|c|}
\hline $\begin{array}{l}\text { Chain } \\
\text { length }\end{array}$ & 6 & & & & 12 & & & & 24 & & & \\
\hline $\begin{array}{l}\text { Number } \\
\text { of chains }\end{array}$ & 5 & 25 & 50 & 100 & 5 & 25 & 50 & 100 & 5 & 25 & 50 & 100 \\
\hline $\begin{array}{l}\text { Job CPU } \\
\text { time } \\
\text { (minutes) }\end{array}$ & 14.95 & 74.75 & 149.5 & 299.0 & 39.8 & 199.0 & 398.0 & 796.0 & 86.35 & 431.75 & 863.5 & 1727.0 \\
\hline $\begin{array}{l}\text { RMSE } \\
\text { polynomi } \\
\text { al (eV) }\end{array}$ & 0.154 & 0.061 & 0.059 & 0.061 & 0.046 & 0.041 & 0.039 & 0.039 & 0.028 & 0.025 & 0.026 & 0.025 \\
\hline $\begin{array}{l}\text { RMSE } \\
\text { linear }\end{array}$ & 0.065 & 0.070 & 0.065 & 0.068 & 0.044 & 0.042 & 0.039 & 0.041 & 0.027 & 0.027 & 0.029 & 0.027 \\
\hline
\end{tabular}

(eV)

Table S3: RMSE values with polynomial and line regression fits for PDPP2TT against time cost of generating the calibration sample (chain length=calibration chain length, number of chains=calibration sample size)

\begin{tabular}{|c|c|c|c|c|c|c|c|c|c|c|c|c|}
\hline $\begin{array}{l}\text { Chain } \\
\text { length }\end{array}$ & 6 & & & & 12 & & & & 24 & & & \\
\hline $\begin{array}{l}\text { Number } \\
\text { of chains }\end{array}$ & 5 & 25 & 50 & 100 & 5 & 25 & 50 & 100 & 5 & 25 & 50 & 100 \\
\hline $\begin{array}{l}\text { Job CPU } \\
\text { time } \\
\text { (minutes) }\end{array}$ & 31.3 & 156.3 & 312.6 & 625.2 & 73.7 & 368.4 & 736.8 & 1473.5 & 239.2 & 1195.8 & 2391.5 & 4783.0 \\
\hline $\begin{array}{l}\text { RMSE } \\
\text { polynomi } \\
\text { al }(e V)\end{array}$ & 0.108 & 0.110 & 0.110 & 0.111 & 0.055 & 0.058 & 0.057 & 0.057 & 0.020 & 0.023 & 0.025 & 0.024 \\
\hline $\begin{array}{l}\text { RMSE } \\
\text { linear }\end{array}$ & 0.111 & 0.115 & 0.117 & 0.116 & 0.051 & 0.056 & 0.054 & 0.056 & 0.020 & 0.024 & 0.025 & 0.024 \\
\hline
\end{tabular}

(eV) 


\section{S4. Density of states (DOS) calculation and error in the DOS post-calibration}

A comparison was done between the density of states (DOS) calculated using the energies from LMOM and exact calculation at B3LYP/3-21G* which is shown for the 5 polymers IDTBT, DPPBTZ, DPPDTT, polythiophene and PDPP2TT in figure S4. The DOSs were obtained for each polymer using energies calculated for samples with 100 chains of 48-mer chains, and with a broadening $\sigma$ of $0.03 \mathrm{eV}$. The calibration was done as described in section 2.4 of the main paper and supplementary information S3, with 5 chains of 12 -mer chains for each polymer as the calibration sample.

The match between these calculations was measured by the differences in features of the DOS near the valence band edge; the energy at the onset of the tail peak (DOS tail onset), the energy at which the tail edge occurs (DOS tail edge), and the difference between the various aforementioned quantities from the LMOM and exact DOS. All these quantities are quoted in table S4. The error of our method is measure by the differences between the LMOM and exact calculation for the position of the DOS tail onset and tail edge. Relative to the corresponding quantities, the error is of the order $10^{-}$ ${ }^{1} \mathrm{eV}$ for the tail width, and of the order $10^{-2} \mathrm{eV}$ for the DOS tail onset and tail edge positions.

\begin{tabular}{|c|c|c|c|c|c|}
\hline Polymer & IDTBT & DPPBTz & DPPDTT & $\begin{array}{l}\text { Polythiophene } \\
\text { (P3HT) }\end{array}$ & PDPP2TT \\
\hline Exact DOS tail edge (eV) & -4.67 & -4.95 & -5.00 & -4.88 & -5.02 \\
\hline $\begin{array}{l}\text { LMOM DOS tail edge } \\
\text { (eV) }\end{array}$ & -4.64 & -4.89 & -4.97 & -4.85 & -4.96 \\
\hline $\begin{array}{l}\text { Absolute difference at } \\
\text { tail edge }(\mathrm{eV})\end{array}$ & 0.03 & 0.06 & 0.03 & 0.03 & 0.06 \\
\hline $\begin{array}{l}\text { Exact DOS tail onset } \\
(\mathrm{eV})\end{array}$ & -4.82 & -5.09 & -5.17 & -5.10 & -5.15 \\
\hline $\begin{array}{l}\text { LMOM DOS tail onset } \\
\text { (eV) }\end{array}$ & -4.81 & -5.04 & -5.17 & -5.09 & -5.11 \\
\hline $\begin{array}{l}\text { Absolute difference at } \\
\text { tail onset (eV) }\end{array}$ & 0.01 & 0.05 & 0.00 & 0.01 & 0.04 \\
\hline
\end{tabular}



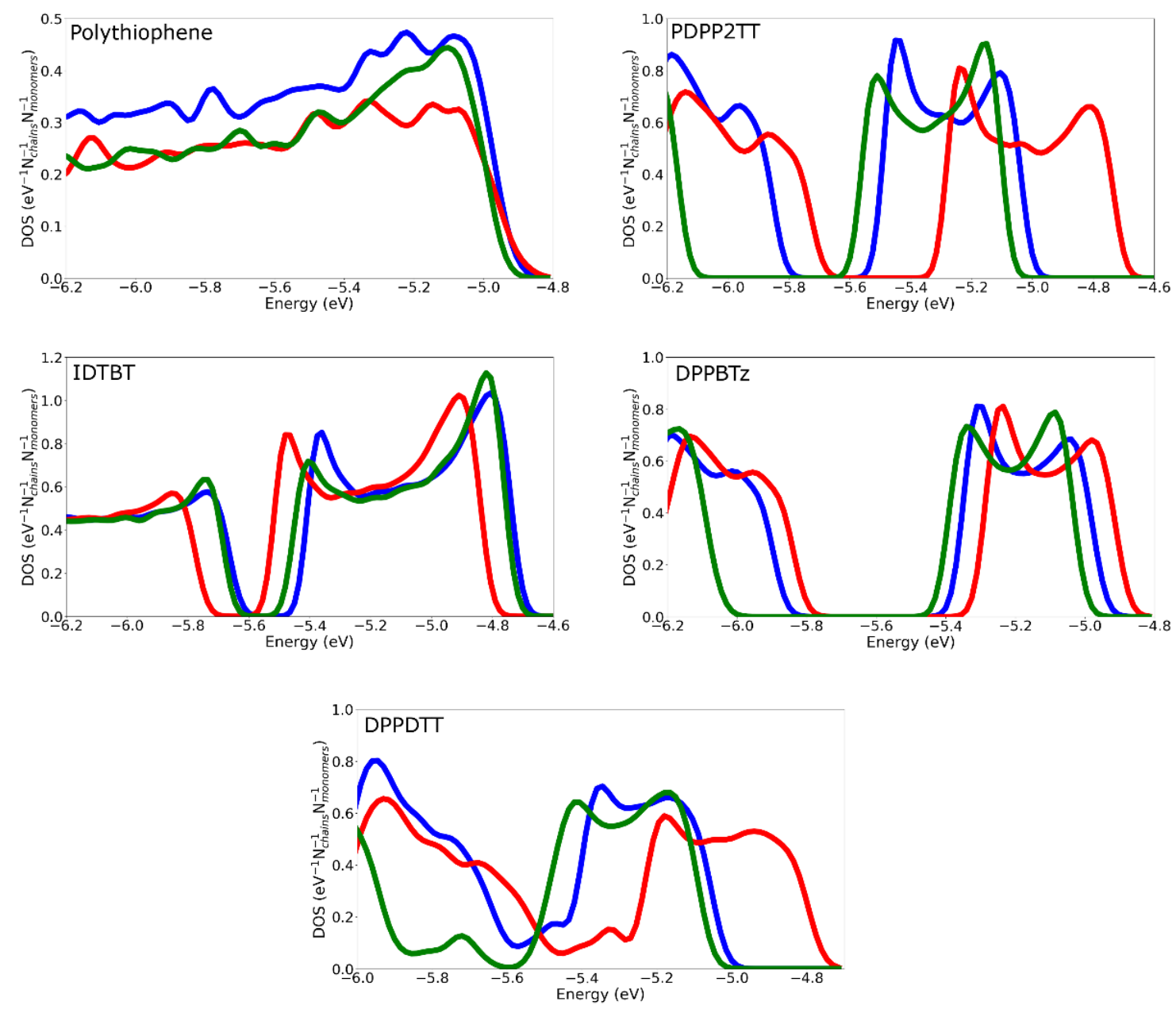

Figure S4: Normalised DOSs calculated using the uncalibrated LMOM energies (red), calibrated LMOM energies (blue) and exact energies (green) for 5 polymers; polythiophene, PDPP2TT, IDTBT, DPPBTz, DPPDTT. The calibrated LMOM energies were obtained with a calibration sample containing 5 different chains of 12-monomer chains, performing the calibration over the $0.5 \mathrm{eV}$ range from the top of the valence band. Structural repeating units (SRUs) of IDT-BT (top), DPP-DTT (middle) and DPP-BTz (bottom) are shown in the inset of each plot.

\section{S5. Calculation of localisation length and inverse participation ratio}

We outline here the method for calculating the inverse participation ratio referred to in section 3 of the main paper. The inverse participation ratio $I P R^{(m)}$ is given by equation SE10, and uses the weight $P_{k}^{(m)}$ of molecular orbital (MO) $m$ on a monomer with index $k$ and the centroid $R^{(m)}$ of chain $\mathrm{MO} m$ calculated using equations 5 and 6 from the main paper, respectively.

$I P R^{(m)}=\left(\sum_{k}\left(P_{k}^{(m)}\right)^{2}\right)^{-1}$

As with the localisation length $L L^{(m)}, I P R^{(m)}$ needs to be expressed in terms of the energy $E$ to be used for ensembles of disorder chains. To do this, the density of states (DOS) $f(E)$ is first calculated as described near the beginning of section 3 in the main paper, then the energy dependent inverse 
participation ratio $\operatorname{IPR}(E)^{(m)}$ of chain MO $m$ (along 1 chain) is obtained as defined in equation SE11, where $E$ is the energy and $E_{m}$ is energy of molecular orbital $\mathrm{m}$.

$\operatorname{IPR}(E)^{(m)}=\frac{\sum_{m} I P R^{(m)} \delta\left(E-E_{m}\right)}{\sum_{m} \delta\left(E-E_{m}\right)}=\frac{\sum_{m} I P R^{(m)} g\left(E-E_{m}\right)}{\sum_{m} g\left(E-E_{m}\right)}$

We calculated the $I P R^{(m)}, I P R(E)^{(m)}, L L^{(m)}$ and $L L(E)^{(m)}$ for the 5 polymers polythiophene, PDPP2TT, IDTBT, DPPDTT and DPPBTz shown in figure S5, where in the calculations we used 100 chains of 48-mer chains for each polymer. In contrast to the main paper, the broadening used for the DOS was $0.015 \mathrm{eV}$ and $L L^{(m)}$ and $L L(E)^{(m)}$ are expressed in unit length instead of Angstroms, thus these instead count the number of monomers participating in an MO like the $\operatorname{IP} R^{(m)}$, and $\operatorname{IPR}(E)^{(m)}$. The $I P R(E)^{(m)}$ and $L L(E)^{(m)}$ both roughly cross the middle point of the bands of $I P R^{(m)}$ and $L L^{(m)}$, represented as scatter points in figure S5. Thus, when expressed in effectively the same units these non-averaged and energy-dependent quantities show similar information with regards to MO localisation. As outlined in section 3 of the main paper, we thus use $L L^{(m)}$ and $L L(E)^{(m)}$ for measuring $\mathrm{MO}$ localisation in the rest of the paper.
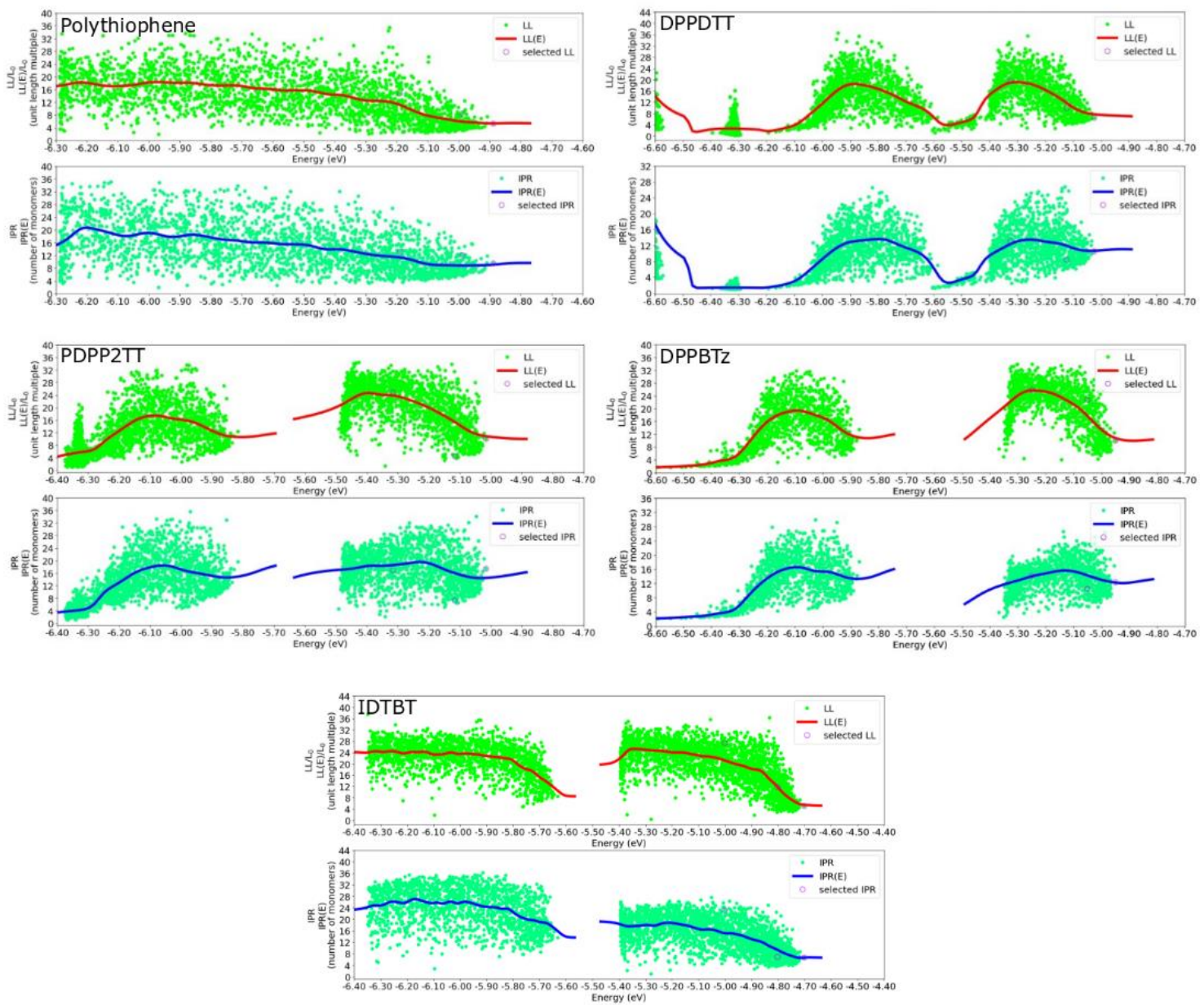

Figure S5: Plots for the $I P R^{(m)}, I P R(E)^{(m)}, L L^{(m)}$ and $L L(E)^{(m)}$ for a set of 5 polymers IDTBT, DPPBTz, DPPDTT, polythiophene and PDPP2TT, with 100 chains of 48-mer chains per polymer calculation. Selected IPR circled were chosen to examine the difference between the energy dependent and normal LLs and IPRs when both were calculated within very close energies. 


\section{S6. Torsional energy vs dihedral angle}

We provide here the details of the torsional energies used to compute the Boltzmann probabilities for selecting the dihedral angles (parameter $\phi_{3}$ ) in polymer chain samples required for DOS calculations, shown in figure $\mathrm{S6}$. These are calculated at the level of theory specified in supplementary information S2. The energies are scaled by subtracting the lowest torsion energy for each dimer, and are thus relative energies. The dimers included are those monomer pairs that appear in the 5 polymers IDTBT, DPPBTz, DPPDTT, polythiophene and PDPP2TT. Additionally, we provide in figure S6 the HOMO to HOMO, HOMO-1 to HOMO-1, and permutations of HOMO to HOMO-1 couplings for the aforementioned dimers plotted against the dihedral angle of the dimer (parameter $\phi_{3}$ ). These couplings are elements taken from the Fock matrix in the LMO basis calculated for each of the dimers.
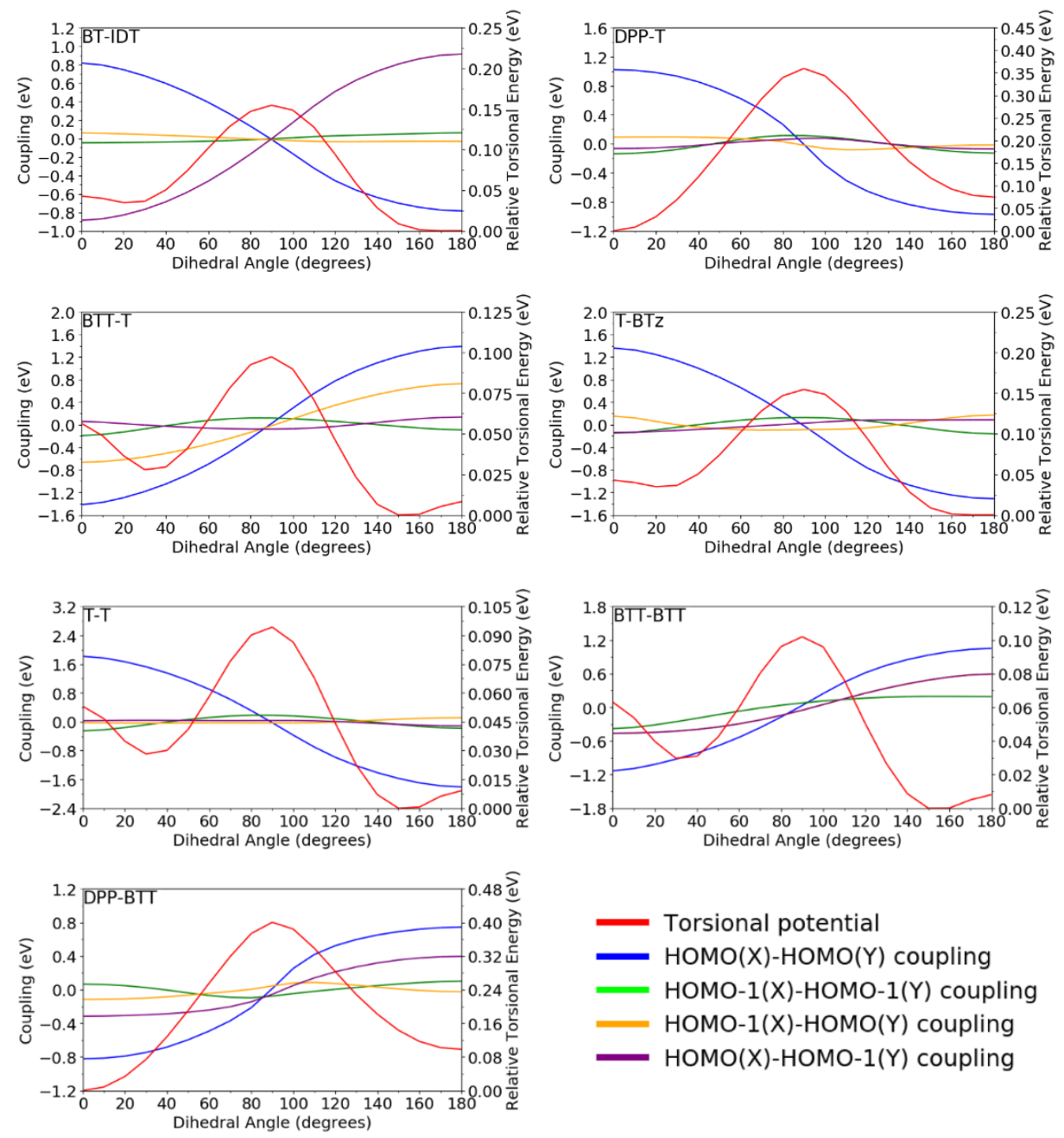

Figure S6: Dihedral angle (parameter $\phi_{3}$ ) vs scaled torsional energy of the monomer pairs that appear in the 5 polymers IDTBT, DPPBTz, DPPDTT, polythiophene and PDPP2TT, with associated HOMO to HOMO, HOMO-1 to HOMO-1, and permutations of HOMO to HOMO-1 couplings. The coupling permutations are distinguished by the variables $X$ and $Y$ in the parentheses, where $X$ and $Y$ are the monomer abbreviated names separated by a hyphen in the top left of each plot; for example, in BTIDT $\mathrm{X}=\mathrm{BT}$ and $\mathrm{Y}=\mathrm{IDT}$.

For each dimer pair in figure $\mathrm{S} 6$ we also provide the dihedral angles (parameter $\phi_{3}$ ) in the range 0$180^{\circ}$, interpolating to 1 degree precision, at the global torsional energy minimum and spread of 
dihedral angles with torsional energy within $k_{B} T(T=298.15 K)$ from the global torsional energy minimum of that dimer. These are listed in table S5.

\begin{tabular}{lll}
\hline Table S5 - Dimer pairs and their most probable dihedral angles \\
\hline Dimer & $\begin{array}{l}\text { Dihedral angle at (interpolated) global } \\
\text { torsional energy minimum (degrees) }\end{array}$ & $\begin{array}{l}\text { Spread of dihedral angles with } \\
\left.\text { torsional energy } \leq \mathrm{k}_{\mathrm{B}} \mathrm{T} \text { ( } \mathrm{T}=298.15 \mathrm{~K}\right) \\
\text { (degrees) }\end{array}$ \\
\hline T-T & 154 & 52 \\
\hline BTT-BTT & 155 & 50 \\
\hline DPP-BTT & 0 & 18 \\
\hline BT-IDT & 169 & 39 \\
\hline DPP-T & 0 & 19 \\
\hline BTT-T & 154 & 52 \\
\hline T-BTz & 169 & 39 \\
\hline
\end{tabular}

\section{S7. Tail slope comparisons and reduced $\pi$-basis set LMOM calculations}

We show a comparison below in figure $S 7$ of the tail slopes at the band edge of the DOS $(E)$ for the 5 polymers IDTBT, DPPBTz, DPPDTT, polythiophene and PDPP2TT. Each DOS(E) is clipped to show only the region in the proximity of the band edge, but are the same as those in figure 8 of the main paper.

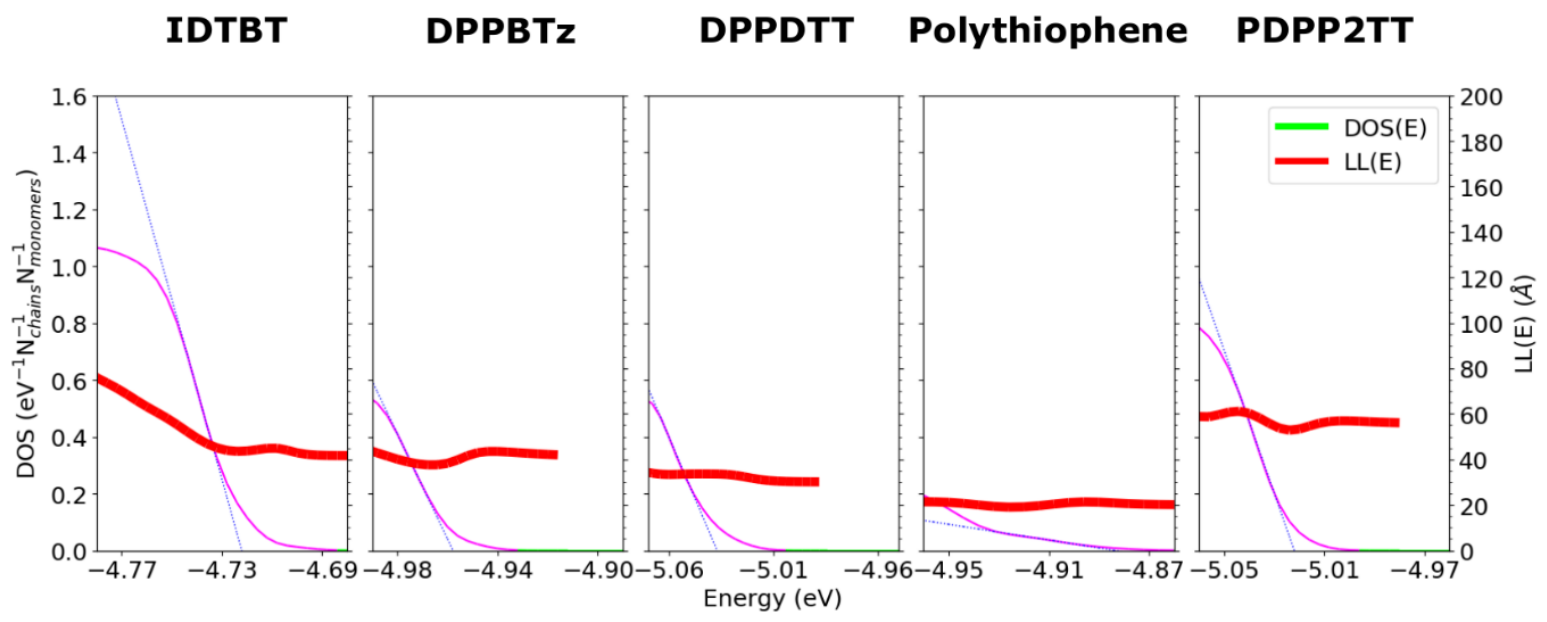

Figure S7: Tail slope comparison at the band edge of the DOSs for the 5 polymers IDTBT, DPPBTz, DPPDTT, polythiophene and PDPP2TT, with slopes shown as dotted blue lines.

The density of states (DOS) and energy dependent localisation length $\left(L L(E)_{E D G E}\right)$ were also calculated with our method using a HOMO basis set for the 5 polymers in our series, and compared with the results from using the full $\pi-\mathrm{MO}$ basis set. The energies in these calculations were obtained using 100 chain samples of 48-mer chains for each polymer and calibrated using the method as described in section 2.4 of the main paper and supplementary information S3, with only the distinction of using a HOMO only basis set to calculate the LMOM energies needed for calibration. The results of these calculations are shown in figure $\mathrm{S} 8$ and table $\mathrm{S} 6$, where the results from the HOMO only and full $\pi-\mathrm{MO}$ basis set calculations are compared in terms of tail width as measured by the steepness of the tail slope and $L L(E)_{E D G E}$ at the band edge. 


\begin{tabular}{|c|c|c|c|c|c|}
\hline Polymer & IDTBT & DPPBTz & DPPDTT & $\begin{array}{l}\text { Polythiophene } \\
\text { (P3HT) }\end{array}$ & PDPP2TT \\
\hline $\begin{array}{l}\text { Tail slope } \\
\left(\mathrm{eV}^{-2} \mathrm{~N}_{\text {chains }}{ }^{-}\right. \\
\left.{ }^{1} \mathrm{~N}_{\text {monomers }}{ }^{-1}\right)\end{array}$ & -27.45 & -15.23 & -20.71 & -1.16 & -28.10 \\
\hline$L L\left(E_{E D G E}\right)(\AA)$ & $59.80 /-4.71$ & $33.76 /-4.97$ & $30.86 /-5.07$ & 18.89/-4.91 & $50.64 /-5.06$ \\
\hline
\end{tabular}

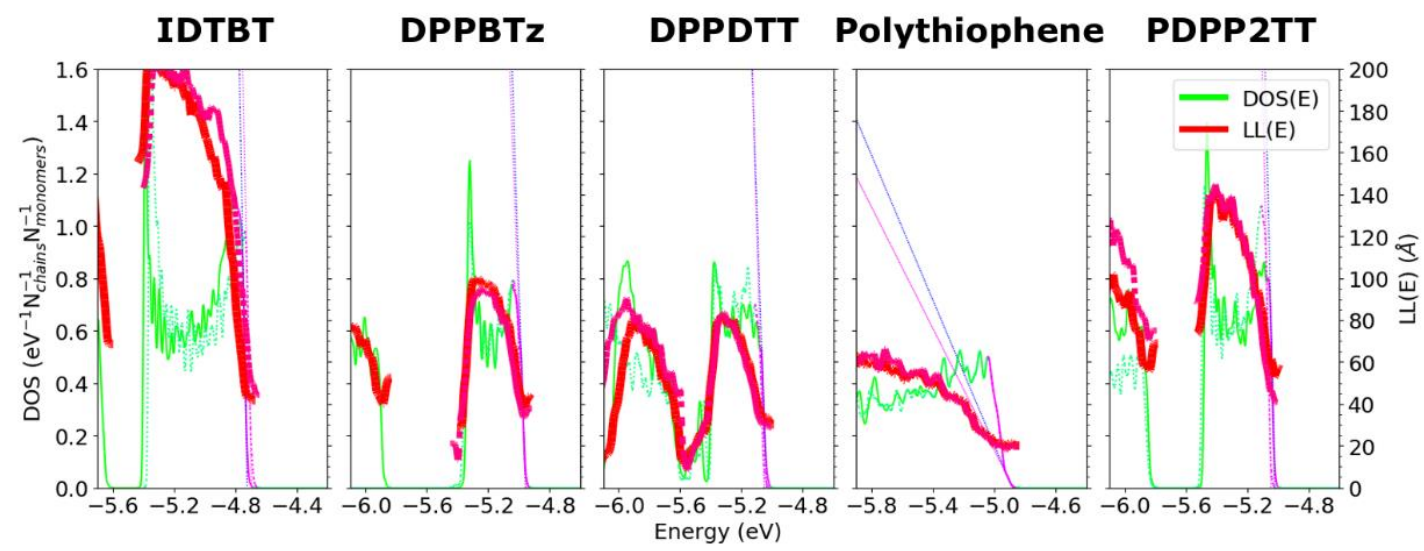

Figure S8: DOS and LL(E) plots for IDTBT, DPPBTz, DPPDTT, polythiophene and PDPP2TT from LMOM calculations with full $\pi-\mathrm{MO}$ basis set (red and green plots) and HOMO only basis set (blue shifted red and green plots) with DOS tail slopes on dotted lines (blue for full $\pi-\mathrm{MO}$ basis set calculations and purple for HOMO only basis set calculations). 